\title{
PARAMETRIC BLIND IMAGE DEBLURRING WITH GRADIENT BASED SPECTRAL KURTOSIS MAXIMIZATION
}

\author{
AFTAB KHAN ${ }^{\bowtie, 1}$ AND HUJUN YIN $^{2}$ \\ ${ }^{1}$ Department of Computer Systems Engineering (DCSE), University of Engineering and Technology (UET), \\ 25000 Peshawar, Pakistan.; ${ }^{2}$ School of Electrical and Electronic Engineering, The University of Manchester, \\ Manchester M13 9PL, UK. \\ e-mail: aftab.khan@uetpeshawar.edu.pk, hujun.yin@manchester.ac.uk \\ (Received January 12, 2018; revised September 9, 2018; accepted September 17, 2018)
}

\begin{abstract}
Blind image deconvolution/deblurring (BID) is a challenging task due to lack of prior information about the blurring process and image. Noise and ringing artefacts resulted during the restoration process further deter fine restoration of the pristine image. These artefacts mainly arise from using a poorly estimated point spread function (PSF) combined with an ineffective restoration filter. This paper presents a BID scheme based on the steepest descent in kurtosis maximization. Assuming uniform blur, the PSF can be modelled by a parametric form. The scheme tries to estimate the blur parameters by maximizing kurtosis of the deblurred image. The scheme is devised to handle any type of blur that can be framed into a parametric form such as Gaussian, motion and out-of-focus. Gradients for the blur parameters are computed and optimized in the direction of increasing kurtosis value using a steepest descent scheme. The algorithms for several common blurs are derived and the effectiveness has been corroborated through a set of experiments. Validation has also been carried out on various real examples. It is shown that the scheme optimizes on the parameters in a close vicinity of the true parameters. Results of both benchmark and real images are presented. Both full-reference and non-reference image quality measures have been used in quantifying the deblurring performance. The results show that the proposed method offers marked improvements over the existing methods.
\end{abstract}

Keywords: blind image deblurring (BID), gradient descent, image quality measures (IQMs), image restoration, kurtosis.

\section{INTRODUCTION}

Since the first realization of blind image deconvolution/ deblurring (BID) in early 1960s, it still remains a challenging task to find an efficient, reliable and most importantly, diversely applicable restoration scheme. A scheme should not only estimate the actual point spread function (PSF) but also restore the image. The main challenge arises from little or no prior information about the image or the blurring process as well as lack of optimal restoration filters that can eliminate the blurring effect. The two common side effects of deblurring include noise amplification and ringing artefacts in the deblurred image. Developing a scheme that can handle different types of blur especially for real images is yet to be achieved to a satisfactory extent.

A variety of BID schemes and restoration filters have been proposed over the years, ranging from the spatial domain to the frequency domain, parametric to non-parametric. Typical methods include the Richardson-Lucy method, total variation, Wiener filter, maximum likelihood method, minimum entropy deconvolution, recursive inverse filter, simulated annealing, and multi-channel blind deconvolution
(Richardson, 1972; Wiggins, 1978; Lagendijk et al., 1988; 1990; Kundur and Hatzinakos, 1996; Banham and Katsaggelos, 1997; Chan and Wong, 1998), as well as their recent improvements (Chen and Cheng, 2011; Szolgay and Szirányi, 2011; Wang and Li, 2011; Yang and Liu, 2011). These methods do provide, to some extent, solutions to the BID problem; however, many are not satisfactory in terms of robustness and performance. The robustness of these schemes becomes questionable when real-life blurred images are to be restored. This is because unlike synthetic deblurring, real deblurring often suffers from noise and ringing effects. The blurred images may have been degraded by arbitrarily shaped PSFs that are complex and cannot be easily modelled (Gupta et al., 2010; Harmeling et al., 2011; Lu, 2012; Whyte et al., 2011; Yuquan et al., 2013).

Recently some BID schemes focus mainly on arbitrary motion blur. Not only because the problem has common occurrence in image acquisition but also because it is challenging. This type of blur ensues from camera shakes or movements of objects or background in the focal range. The PSFs of such blur are usually of arbitrary shape and are sometimes space variant. Restoring such images requires more effort and 
complex procedures are applied to approximate the blur kernel as well as restore the image while deterring deblurring noise and ringing artefacts. Restoring motion blurred images as compared to Gaussian and out-of-focus blurred ones is less problematic in restoration terms as for other types more ringing artefacts and significant amount of residual blur are often produced.

The image processing community and researchers over the years have strived to come up with BID schemes that can effectively handle different types of blurs. Our motivation is to design a BID scheme that is efficient in terms of computational costs and effective in terms of restoration quality. The proposed scheme uses a parametric representation for uniform blur. This reduces the search space for PSF parameters. The parameters are optimized by a steepest descent scheme on a restoration measure. The method offers a unified framework and can estimate any parametric blur type.

\section{METHODS}

In this following sub-sections, the various concepts used in implementing our proposed scheme are discussed. It includes the introduction and modelling of parametric blurs, the principle of kurtosis maximization and the gradient descent scheme based on this principle.

\section{PARAMETRIC BLUR MODELS}

Parametric models help simplify the search for the blur function, while a restoration measure is used to aid the optimization to a desired result. In the quest for a simple deblurring scheme, the proposed method relies on higher order statistics based non-Gaussianity measure to lead in the direction of increased quality of the deblurred image. Previously the authors have proposed schemes using non-Gaussianity measures in either spatial or frequency domain, namely spatial and spectral kurtosis (Yin and Hussain, 2008; Khan and Yin, 2011). In addition, re-blurring based image quality measure was also investigated but its use was limited as it is highly sensitive to restoration noise and artefacts.

Let $x(m, n)$ represent the original image without any form of degradation, $h(m, n)$ be the PSF, and $y(m, n)$ the output of the image acquisition system. For a system of stationary impulse response across the entire image (i.e. a spatially invariant PSF), the convolution is given by (Oppenheim et al., 1968; Sondhi, 1972),

$$
y(m, n)=h(m, n) * f(m, n)+\eta(m, n),
$$

where $*$ denotes the convolution operator, $\eta(m, n)$ represents additive noise; and $m$ and $n$ are spatial coordinates. The frequency domain representation of Eq. (1), with spectral coordinates $i$ and $j$, is given as

$$
Y(i, j)=H(i, j) F(i, j)+\prod(i, j) .
$$

Parametric blur models are discusses as follows.

The blur PSFs studied here are spatially invariant. Modelling, restoration and estimation of the original image from invariant PSFs is less cumbersome as compared to spatially variant ones. The classes of blur that can be easily modelled include atmospheric turbulence, motion and out-of-focus. The PSF for atmospheric turbulence blur for long exposures can be approximated by Gaussian blur and the degraded image regarded as a Gaussian blurred image (Lagendijk and Biemond, 2009). The 2-D Gaussian $\mathrm{PSF}$ is given by

$$
h(m, n)=\frac{1}{2 \pi \sigma^{2}} \exp \left(-\frac{m^{2}+n^{2}}{2 \sigma^{2}}\right),
$$

where $\sigma$ is the width of the blurring kernel.

Motion blurs commonly result from relative motions between the scene and the recording device. They can be in the form of translation, sudden change in scale, rotation or a combination of these. The motion blur dealt with here is the case of linear translation. Denoting the length of motion by $L$ and the angle by $\varphi$, the motion blur PSF is given as (Lagendijk and Biemond, 2009),

$$
h(m, n ; L, \varphi)=\left\{\begin{array}{cc}
1 / L & \text { if } \sqrt{m^{2}+n^{2}} \leq L / 2 \\
0 & \text { and } m / n=-\tan \varphi \\
\text { elsewhere }
\end{array}\right.
$$

When a camera captures a 2-D image of a 3-D scene, certain points in the scene may not be focused. For a camera with a circular aperture, the PSF of a point looks like a circular disk with a specific radius. The spatially continuous out-of-focus blur of radius $R$ can be expressed as (Lagendijk and Biemond, 2009),

$$
h(m, n ; R)= \begin{cases}\left(C \pi R^{2}\right)^{-1} & \text { if } \sqrt{m^{2}+n^{2}} \leq R^{2}, \\ 0 & \text { elsewhere }\end{cases}
$$

where $C$ is a constant and is chosen so that energy conservation law is satisfied. 


\section{KURTOSIS MAXIMIZATION}

If statistical independence can be assumed for the unknown source signals, then according to the Central Limit Theorem (CLT) the output signals of a linear system are more Gaussian. Hence a general BID scheme can be designed to estimate the independent signals that are maximally non- Gaussian. A measure of non-Gaussianity such as kurtosis or negentropy can be employed to achieve this goal. The normalized kurtosis of an image $y$ is defined as

$$
K(y)=\frac{E\left\{y^{4}\right\}}{\left(E\left\{y^{2}\right\}\right)^{2}}-3 .
$$

Using a suitable optimization scheme, the kurtosis can be optimized in the direction of maximum non-Gaussianity for the deblurred image. However, optimizing the kurtosis function is not a trivial task and resulting learning algorithm can be difficult to implement or may induce high computational costs. The proposed scheme utilizes the Wiener filter to estimate the deblurred image for a candidate PSF. Kurtosis calculated for the spatial domain, referred to as spatial kurtosis, is computationally costly because its calculation on the deblurred image needs to be transformed from the frequency domain back to the spatial domain in each iteration. By calculating the non-Gaussianity of the spectrum images, this overhead is drastically reduced. For this purpose, the kurtosis of an image is calculated using the spectral kurtosis (Khan and Yin, 2011) defined for the frequency domain image, $Y$, as

$$
K(Y)=\frac{E\left\{Y^{4}\right\}}{\left(E\left\{Y^{2}\right\}\right)^{2}}-3 .
$$

Even so, the previous work (Yin and Hussain, 2008) had used a genetic algorithm to search for optimal blur parameters. The process is often time consuming and there is no guarantee that an optimal solution will be reached. Based on the statistical nature of the image, i.e. super-Gaussian or sub-Gaussian, spatial kurtosis is either minimized or maximized at the correct blur parameters. However, in the case of spectral kurtosis, where magnitude spectrum is used,the maximum is always reached for the deblurred image with the correct parameters. A new gradient based BID scheme that utilizes parametric blur models and spectral kurtosis as the non-Gaussianity measure is proposed in this research work.

\section{IMAGE QUALITY MEASURES}

The proposed scheme has been tested on a variety of images, synthetic and real blurred, degraded with various functions. A number of image quality measures were employed to evaluate the quality of deblurred images. The full reference Image Quality Assessment (IQA) measures include the Mean Structural SIMilarity (MSSIM) index (Wang et al., 2004; Wang and Li, 2011; Brunet et al., 2012) and the Universal Quality Index (UQI) (Wang and Bovik, 2002). The blind/no-reference based IQA measures are the Blind/Reference-less Image Spatial QUality Evaluator (BRISQUE) (Mittal et al., 2011; 2012b) and the Natural Image Quality Evaluator (NIQE) (Mittal et al., 2012a; 2013). These quality measures are briefly described below.

The SSIM is an objective image quality metric that measures the structural similarity between two images by comparing local patterns of pixel intensities normalized for luminance and contrast. Given two images, $f$ and $g$, the SSIM is a function of luminance $l(f, g)$, contrast $c(f, g)$ and structural similarity $s(f, g)$.

$$
\begin{aligned}
\operatorname{SSIM}(f, g) & =t(l(f, g), c(f, g), s(f, g)), \\
l(f, g) & =\frac{2 \mu_{f} \mu_{g}+C 1}{\mu_{f}^{2}+\mu_{g}^{2}+C 1}, \\
c(f, g) & =\frac{2 \sigma_{f} \sigma_{g}+C 2}{\sigma_{f}^{2}+\sigma_{g}^{2}+C 2}, \\
s(f, g) & =\frac{\sigma_{f g}+C 3}{\sigma_{f} \sigma_{g}+C 3},
\end{aligned}
$$

where $\mu$ and $\sigma$ are the mean and the standard deviation of the image, respectively; $C 1, C 2$ and $C 3$ are constants; and $t$ represents the SSIM transfer function. SSIM is computed at each pixel and generally its mean value is used, so named Mean SSIM (MSSIM). SSIM has been used for denoising and classification (Gao et al., 2011; Rehman and Wang, 2011).

The UQI analyzes the loss of correlation, luminance and contrast distortion among the two images as the base for quality perception. The UQI for two images, $f$ and $g$, is defined as (Wang and Bovik, 2002),

$$
\begin{aligned}
& Q(f, g)=\frac{4 \sigma_{f g} \mu_{f} \mu_{g}}{\left(\sigma_{f}^{2}+\sigma_{g}^{2}\right)\left(\mu_{f}^{2}+\mu_{g}^{2}\right)}, \\
& Q(f, g)=\frac{\sigma_{f g}}{\sigma_{f} \sigma_{g}} \cdot \frac{2 \mu_{f} \mu_{g}}{\mu_{f}^{2}+\mu_{g}^{2}} \cdot \frac{2 \sigma_{f} \sigma_{g}}{\sigma_{f}^{2}+\sigma_{g}^{2}} .
\end{aligned}
$$

The first term is the correlation coefficient of the two images whereas the second and third terms are the measures of mean luminance and structural similarities.

The BRISQUE measures distortion based on spatial domain statistics. It is also a non-reference IQA model. Rather than computing distortion specific 
features such as ringing, blur or blocking, it uses scene statistics of locally normalized luminance coefficients to quantify possible losses of naturalness in the image due to the presence of distortions, thereby leading to a holistic measure of quality. The BRISQUE uses a mapping from feature space to quality scores with a regression module that yields a measure of image quality. The features comprise statistic measures of a generalized Gaussian distribution fitting of the Mean Subtracted Contrast Normalized (MSCN) coefficients. The MSCN for the distorted image $g$ is obtained by subtracting the local mean signal value $\mu_{g}$ and then dividing by the local contrast function $\sigma_{g}$ such that

$$
\hat{g}(m, n)=\frac{g(m, n)-\mu_{g}(m, n)}{\sigma_{g}(m, n)+C},
$$

where

$$
\begin{aligned}
\mu_{g}(m, n) & =\sum_{k=-U}^{U} \sum_{l=-V}^{V} w_{k, l} g_{k, l}(m, n), \\
\sigma_{g}(m, n) & =\sqrt{\sum_{k=-U}^{U} \sum_{l=-V}^{V} w_{k, l}\left(g_{k, l}(m, n)-\mu_{g}(m, n)\right)^{2}}
\end{aligned}
$$

where $C$ is a constant, and $w=\left\{w_{k, l} \mid k=-U, \ldots, U, l=\right.$ $-V, \ldots, V\}$ is a 2-D circular-symmetric Gaussian weighting function and $U=V=3$ was used for calculating the measure.

The non-reference NIQE is a blind image quality analyzer that only makes use of measurable deviations from statistical regularities observed in natural images, without being trained on human-rated distorted images and without any exposure to distorted images. The same computation for image quality as in the BRISQUE is used with the exception that NIQE uses natural scene statistics features from a staple of natural images, while the BRISQUE is trained on features obtained from both natural and distorted images as well as human judgment of quality of the images.

\section{IMAGE DATASET}

Images from the Desktop Nexus image wallpaper database (Nexus, 2010) were used in the experiments in the synthetic blur case. Real blurred natural images, captured by either ourselves or others, were used in deblurring real blind images in the experiments.

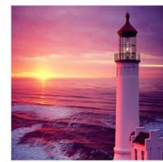

Img-01

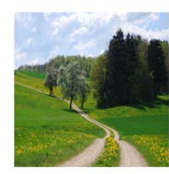

Img-05

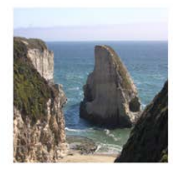

Img-09

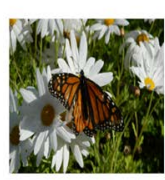

Img-13

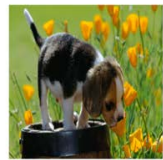

Img-02

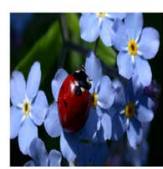

Img-06

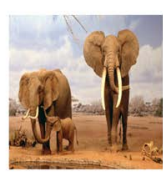

Img-10

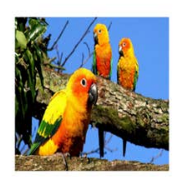

Img-14

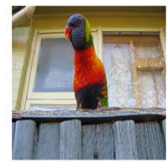

Img-03

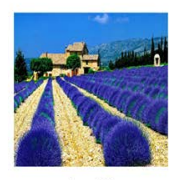

Img-07

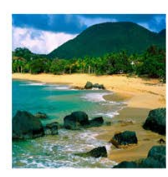

Img-11

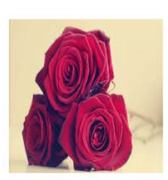

Img-15

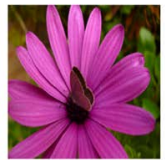

Img-04

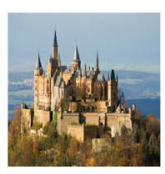

Img-08

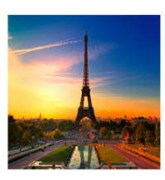

Img-12

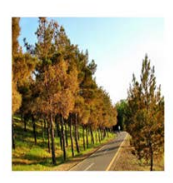

Img-16
Fig. 1. Test images used for deblurring of synthetic blurred images.

A new gradient based BID scheme that utilizes parametric blur models and spectral kurtosis as the non-Gaussianity measure is proposed next.

\section{PROPOSED GRADIENT BASED DEBLURRING SCHEME}

The derivation of the gradient based BID scheme starts with differentiation of the cost function with respect to the parameter of the blurring PSF model. A Kurtosis based cost function for the deblurred image is employed. The parameter is updated iteratively using the steepest descent,

$$
\lambda_{p+1}=\lambda_{p}+\alpha \nabla\left|K_{\lambda_{p}}(\hat{X})\right|
$$

under the constraint,

$$
\left|K_{\lambda_{p+1}}(\hat{X})\right|>\left|K_{\lambda_{p}}(\hat{X})\right|,
$$

where $\lambda$ is a blur PSF parameter, such as kernel width, length or radius in case of Gaussian, motion or outof-focus blur respectively; $p$ is the iteration number; $K_{\lambda_{p}}(\hat{X})$ is the spectral kurtosis of the deblurred image for the current value of $\lambda . \nabla\left|K_{\lambda_{p}}(\hat{X})\right|$ represents the gradient matrix for the magnitude of the complex kurtosis variable and $\alpha$ is the step size. Since

$$
\left|K_{\lambda}(\hat{X})\right|=K_{\lambda}(\hat{X}) \operatorname{sign}\left(K_{\lambda}(\hat{X})\right)
$$


Eq. (8) can be rewritten as

$$
\lambda_{p+1}=\lambda_{p}+\alpha \nabla K_{\lambda_{p}}(\hat{X}) \operatorname{sign}\left(K_{\lambda_{p}}(\hat{X})\right) .
$$

For $\alpha$, a fixed scalar value can be used, $0<\alpha \leq$ 1. For faster convergence, more efficient computation schemes can also be used such as the Newton method or conjugate gradient. As $\hat{X}$ is the deblurred image resulting from deconvolution of the blurred image with the Wiener restoration filter, which is given by

$$
\frac{\hat{H}^{*}}{|H|^{2}+\delta},
$$

where $\delta$ is the noise to signal ratio. For simplicity of future derivations and considering the magnitude of the complex data, we rewrite the equation as

$$
\frac{\hat{H}}{\hat{H}} \frac{\hat{H}^{*}}{|\hat{H}|^{2}+\delta}=\left(\frac{\hat{H} \hat{H}^{*}}{|\hat{H}|^{2} \hat{H}+\hat{H} \delta}\right)=\frac{\hat{H}^{2}}{\hat{H}^{3}+\hat{H} \delta} .
$$

The kurtosis of the deblurred image $\hat{X}$ is given by

$$
K(\hat{X})=\frac{\mu_{\hat{X}}(4)}{\mu_{\hat{X}}(2)^{2}}-3,
$$

where $\mu_{\hat{X}}(k)$ is the $k^{\text {th }}$ central moment. Let $\mu_{\hat{X}}(0)$ represent the expected value or mean of $\hat{X}$.

$$
\mu_{\hat{X}}(0)=\frac{1}{M-Q_{1}} \frac{1}{N-Q_{2}} \sum_{i=Q_{1}+1}^{M} \sum_{j=Q_{2}+1}^{N} \hat{X}(i, j) .
$$

$Q_{1}$ and $Q_{2}$ are regions of uniform convolution, while $M$ and $N$ are image rows and columns respectively. The gradient matrix is derived as follows:

$$
\nabla K_{\lambda}(\hat{X})=\frac{\partial}{\partial \lambda} K(\hat{X})=\frac{\partial}{\partial \lambda}\left(\frac{\mu_{\hat{X}}(4)}{\mu_{\hat{X}}(2)^{2}}\right) .
$$

Expanding the cumulants, we get

$$
\begin{aligned}
& \nabla K_{\lambda}(\hat{X})= \\
& \frac{\partial}{\partial \lambda}\left[\left(\frac{1}{M-Q_{1}} \frac{1}{N-Q_{2}} \cdot\right.\right. \\
& \left.\quad \sum_{i=Q_{1}+1, j=Q_{2}+1}^{M, N}\left[\hat{X}(i, j)-\mu_{\hat{X}}(0)\right]^{4}\right) / \\
& \quad \frac{1}{M-Q_{1}} \frac{1}{N-Q_{2}} \cdot \\
& \left.\left.\sum_{i=Q_{1}+1, j=Q_{2}+1}^{M, N}\left[\hat{X}(i, j)-\mu_{\hat{X}}(0)\right]^{2}\right)^{2}\right] .
\end{aligned}
$$

$$
\begin{aligned}
& \nabla K_{\lambda}(\hat{X})= \\
& 4\left(\frac{1}{\mu_{\hat{X}}(2)^{2}} \frac{1}{M-Q_{1}} \frac{1}{N-Q_{2}} \cdot\right. \\
& \left.\sum_{i=Q_{1}+1}^{M} \sum_{j=Q_{2}+1}^{N} \eta\left[\hat{X}(i, j)-\mu_{\hat{X}}(0)\right]^{3}\right)- \\
& 4\left(\frac{\mu_{\hat{X}}(4)}{\mu_{\hat{X}}(2)^{3}} \frac{1}{M-Q_{1}} \frac{1}{N-Q_{2}} .\right. \\
& \left.\sum_{i=Q_{1}+1}^{M} \sum_{j=Q_{2}+1}^{N} \eta\left[\hat{X}(i, j)-\mu_{\hat{X}}(0)\right]\right),
\end{aligned}
$$

where

$$
\eta=\frac{\partial}{\partial \lambda}\left(\hat{X}-\mu_{\hat{X}}(0)\right)=\frac{\partial}{\partial \lambda}(\hat{X})-\frac{\partial}{\partial \lambda}\left(\mu_{\hat{X}}(0)\right) .
$$

$\partial\left(\mu_{\hat{X}}(0)\right) / \partial \lambda$ is equivalent to the mean value of $\partial \hat{X} / \partial \lambda$ and

$$
\begin{aligned}
\frac{\partial}{\partial \lambda} \hat{X} & =\frac{\partial}{\partial \lambda}\left(G\left(\frac{\hat{H}^{2}}{\hat{H}^{3}+\hat{H} \delta}\right)\right) \\
& =G \frac{\partial}{\partial \lambda}\left(\frac{\hat{H}^{2}}{\hat{H}^{3}+\hat{H} \delta}\right) .
\end{aligned}
$$

Through further manipulations, we get

$$
\begin{aligned}
& \frac{\partial}{\partial \lambda} \hat{X}= \\
& \quad G\left(\frac{2 \hat{H}}{\hat{H}^{3}+\hat{H} \delta}+\hat{H}^{2}\left(\frac{-\left(3 \hat{H}^{2}+\delta\right)}{\left(\hat{H}^{3}+\hat{H} \delta\right)^{2}}\right)\right) \frac{\partial}{\partial \lambda} \hat{H} .
\end{aligned}
$$

The term $\partial \hat{X} / \partial \lambda$ needs to be calculated when a specific type of blur is used. As an example, the derivation for the Gaussian blur PSF is as follows.

For Gaussian blur, the Optical Transfer Function (OTF) for the parametric model is given by

$$
\begin{gathered}
\hat{H}(i, j)=\frac{1}{2 \pi \sigma_{f}^{2}} \exp \left(-\frac{i^{2}+j^{2}}{2 \sigma_{f}^{2}}\right), \\
\frac{\partial}{\partial \lambda} \hat{H}(i, j)=\frac{\partial}{\partial \sigma} \hat{H}(i, j)=\frac{\partial}{\partial \sigma}\left(\frac{1}{2 \pi \sigma^{2}} \exp \left(-\frac{i^{2}+j^{2}}{2 \sigma^{2}}\right)\right) \\
=\frac{-2}{2 \pi \sigma^{3}} \mathrm{e}^{\left(-\frac{i^{2}+j^{2}}{2 \sigma^{2}}\right)}+\frac{1}{2 \pi \sigma^{2}} \frac{\partial}{\partial \sigma}\left(\mathrm{e}^{\left(-\frac{i^{2}+j^{2}}{2 \sigma^{2}}\right)}\right) \\
=\hat{H}(i, j)\left(\frac{-2}{\sigma}+\frac{i^{2}+j^{2}}{\sigma^{3}}\right) .
\end{gathered}
$$


The derivations of the gradient matrix for the motion and out-of-focus blur can be found in Appendix A.

The blur parameters of the PSF model are optimized in the direction of increasing value of the absolute kurtosis till the gradient becomes flat or the difference of kurtosis between successive iterations is smaller than a specified tolerance.

\section{RESULTS}

The proposed scheme has been tested on a variety of images, synthetic and real blurred, degraded with various functions. Image quality measures were employed to evaluate the quality of deblurred images. These included the IQA, MSSIM, UQI, BRISQUE and NIQE quality measures. The results for the proposed scheme are presented below.

\section{DEBLURRING SYNTHETIC BLURRED IMAGES}

Fig. 1 depicts the test images from Desktop Nexus image database. Fig. 2 shows the restorations from their Gaussian-blurred counterparts using the proposed scheme.
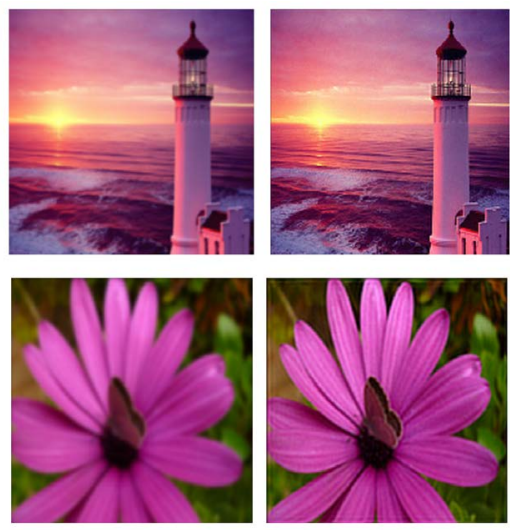

Fig. 2. Gaussian blurred images and their respective estimates using the proposed BID scheme.

The estimated PSF parameters and comparisons of different quality measures for the deblurring are given in Table 1.
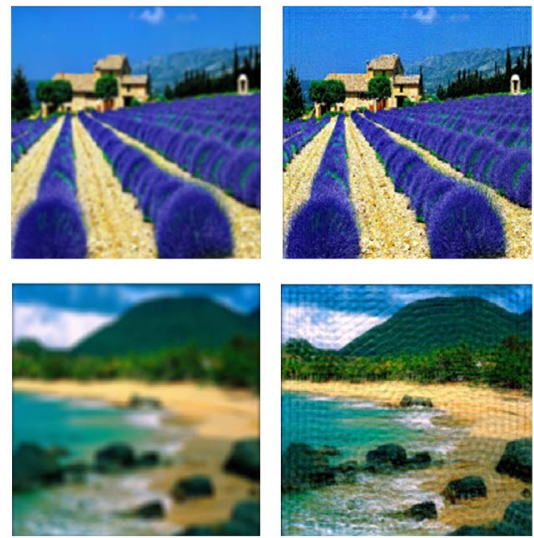

Fig. 3. Out-of-focus blurred images and their respective estimates using the proposed BID scheme.

Fig. 3 illustrates the out-of-focus blurred images and their deblurred counterparts by the proposed scheme. Table 2 summarizes the results of the deblurring along with a comparison by different image quality measures. The deblurring examples for motion blur are shown in Fig. 4, with results summarized in Table 3.

\section{DEBLURRING REAL BLURRED IMAGES}

The proposed deblurring scheme has also been employed to restore real images with motion blur, a typical problem facing photographers. The deblurred images have been compared with the estimated images using the scheme by Shan et al. (2008) and that of Cho and Lee (2009). These two recently proposed schemes have received a great deal of attention due to their advantages.
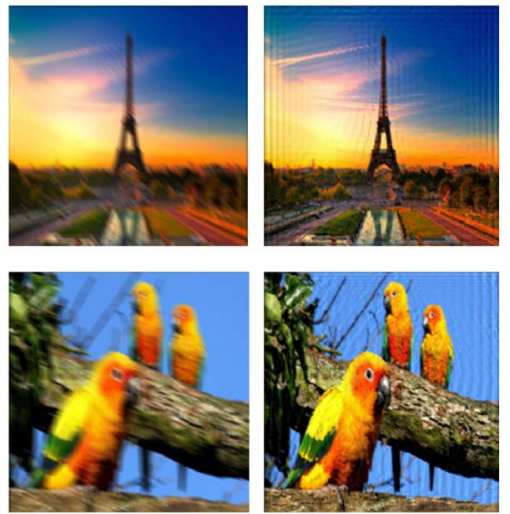

Fig. 4. Motion blurred images and their respective estimates using the proposed BID scheme.

In the first case, an image was captured by a low quality camera. The blurred image, LABEL image, depicted in Fig. 5 (a), shows an approximate vertical motion blur with the small digits at the bottom 
of the image becoming unreadable. The image also contains noise and uneven illumination due to poor lighting one of the most difficult cases in deblurring. Fig. 5(b) shows the deblurred image estimated using the proposed scheme with the estimated blur kernel shown in Fig. 5(e). Using the proposed scheme, a uniform motion PSF of length 21 pixels and angle eight seven degree was estimated. Fig. 5(c) shows the deblurred image and Fig. 5(f) the estimated PSF using the scheme of Shan et al. (Shan et al., 2008). The image in Fig. 5(c) seems to have recovered well; however, the digits are still unreadable since the PSF estimated using this scheme does not completely follow a uniform motion blur.

Fig.5(d) shows the deblurred image with the Cho et al scheme (Cho and Lee, 2009) with the estimated PSF shown in Fig. 5(g). The image appears smoother and the blurring seems to be reasonably diminished. However, the small text in the figure is not fully recovered. Even the relatively larger text in the lower right portion of the image is not clear. This is due to the error in approximation of the blurring PSF especially the angle of the motion blur. In Fig. 5(b), the digits in the deblurred image are much clear and easily readable. An edge-taper function was used to reduce the ringing effect caused by the discrete Fourier Transform.

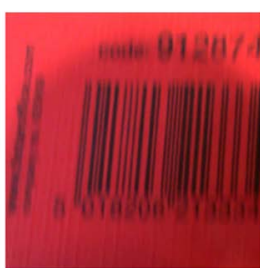

(a)

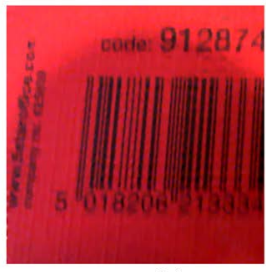

(c)

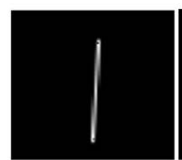

(e)

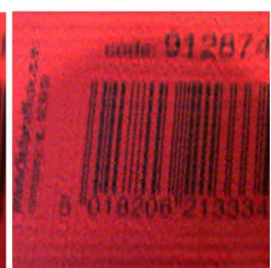

(b)



(d)

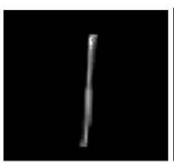

(f)

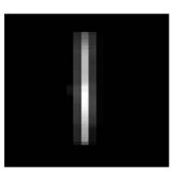

(g)
Fig. 5. (a) Real motion blurred LABEL image. Deblurred images: (b) using the proposed scheme, $(c)$ using Shan et al.s scheme, (d) using Cho et al.s scheme. Estimated PSFs: (e) proposed scheme, (f) Shan et als scheme, ( $g$ ) Cho et al.s scheme.
Fig. 6 and 7 show the deblurring of motion blurred images affected by object movement "MATLAB BOOK" image and camera handshake "BUILDINGS" image. The motion blur was almost linear at a certain angle. Again recovery was achieved to a good extent.

Another motion blurred image taken by an ordinary digital camera and its deblurred versions are shown in Fig. 8. Fig. 8 (b) and (e) shows the deblurred image and the estimated PSF using the proposed scheme. Fig. 8 (c) and (f) show the deblurred image and estimated PSF using the Shan et al scheme. Fig. 8 (d) and (g) show the deblurred image and estimated PSF using the Cho et al. BID scheme. Fig. 8(d) is not recovered well due to the poor estimation of the blurring PSF. The proposed scheme is clearly better and the main and sub title text of the book become more readable.

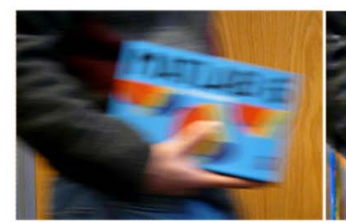

(a)

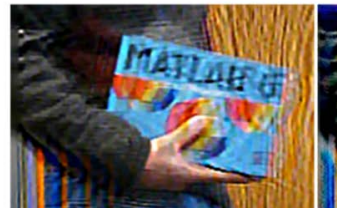

(c)



(b)



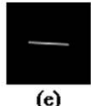

(e)
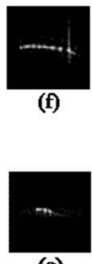

Fig. 6. (a) Real motion blurred MATLAB BOOK image. Deblurred images in (b) using the proposed scheme, (c) using Shan et al. scheme, (d) using Cho et al. scheme; and their respective estimated PSFs in $(e),(f)$ and $(g)$.

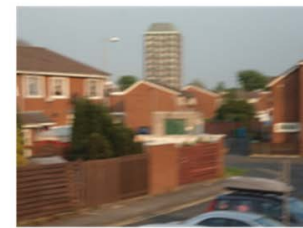

(a)

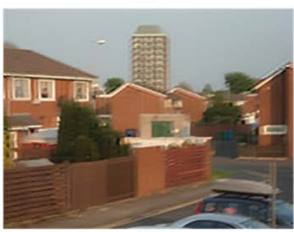

(c)

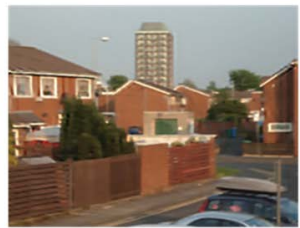

(b)



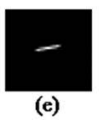
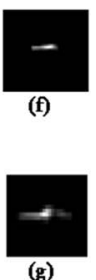

(g)

Fig. 7. (a) Real motion blurred BUILDING image. Deblurred images: (b) using the proposed scheme, $(c)$ using Shan et al. scheme, (d) using Cho et al. scheme; and their respective Estimated PSFs: $(e),(f)$ and $(g)$. 


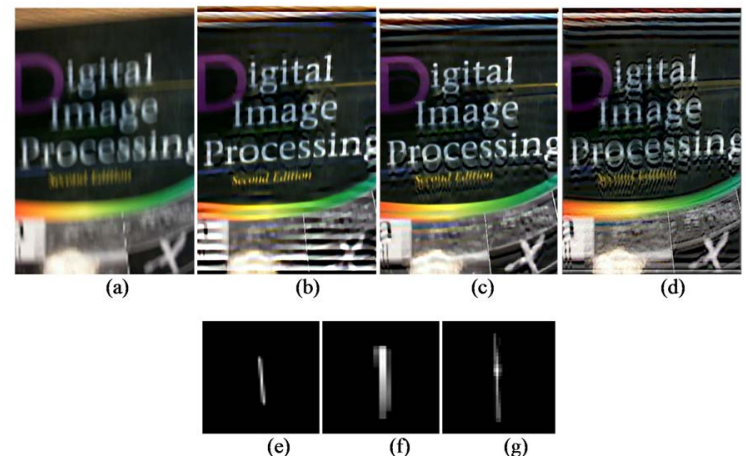

Fig. 8. (a) Real motion blurred DIP BOOK image. Deblurred images: (b) using the proposed scheme, $(c)$ using Shan et al. scheme, (d) using Cho et al. scheme; and their respective Estimated PSFs: $(e),(f)$ and $(g)$.

Table 4 shows the BRISQUE and NIQE measures for the deblurred images in Figs. 5-8. It illustrates the quantitative measures for the deblurred images estimated by these schemes. The BRISQUE and NIQE values for the original, blurred and deblurred images are shown as references and discussion on deblurring qualities. Ideally, the lower values such non-reference measures the higher qualities of the deblurred images. Though the deblurred images in Fig. 5(d), Fig. 6(d) and Fig. 8(d) by Cho et al.'s scheme did not recover well as visually, the BRISQUE and NIQE values seem to depict the deblurred images of high quality. This is due to the over smoothing of the deblurred images by Cho et al.'s scheme in order to recover from the ringing artefacts. In some cases, the original (blurred) images have lower measures than their deblurred version. The tests also show that BRISQUE and NIQE IQMs may not be consistent with blind IQMs and more effort is still required to evaluate their consistency as a performance measure.

\section{DISCUSSION}

In this research work, a new technique for the restoration of blurred images under the influence of parametric blurs is presented. The scheme estimates the blurring parameters by utilising a gradient based kurtosis maximization method for the deblurred image. The proposed method can handle various forms of parametric blurs including Gaussian, motion and out-of-focus (Lagendijk and Biemond, 2009). The proposed method operates on single image and is simple, efficient and easy to implement. Gradients for the blur parameters were computed and optimized in the direction of increasing kurtosis value using a steepest descent scheme. The gradients were derived for several common blurs and the proposed method was tested on artificial and real-life blurs. These two image datasets validated the research work. The results demonstrate the effectiveness of our proposed model.

Firstly, the algorithm was tested on the Desktop Nexus image dataset (Nexus, 2010). This included the generation and recovery of the parametric blurs from their model equations. Uniform blurring was assumed. Afterwards, the images were presented to the steepest descent scheme which estimated the parameters of the blurred. In order to do so, the steepest descent scheme took feedback from the increasing value of kurtosis. this would guide the proposed model in the right direction. Kurtosis is a higher order statistic which in conjunction with the Central Limit Theorem's principle, aids in the recovery of blurred images (Yin and Hussain, 2008).

The robustness of the deblurring schemes become questionable when real-life blurred images need to be restored. Once our proposed scheme was validated for artificial blurring, it was tested on real images captured under the influence of the parametric motion blur. Restoring such images requires more effort as simultaneously approximating the blurring kernel and restoring the image while deterring deblurring noise and ringing artefacts is complex.

Among the various restoration filters, the Wiener filter was opted over the Richardson-Lucy and Total Variation (TV) filter for its ability to recover the image in a single pass and with high deblurring quality. A number of image quality measures were employed to evaluate the quality of deblurred images. This included both full-reference and non-reference image quality measures in quantifying the deblurring performance. Full-reference IQA measures included MSSIM and UQI while the blind/non-reference IQA measures included BRISQUE and NIQE. The proposed scheme is highly efficient as it is independent of a pristine reference image and is able to deblur the images effectively. Hence, the proposed method can be used in deblurring applications where the reference pristine image is not available.

The deblurred images have been compared with the schemes of Shan et al. (2008) and Cho and Lee (2009) and observed that it performs better than the benchmark restoration schemes. It was observed that for both datasets the proposed scheme works better as evident from the full reference and non-reference IQA measures.

\section{CONCLUSIONS}

A blind deblurring is one of the most challenging problem in image restoration. A scheme based on 
Table 1. PSF parameter estimation for Gaussian blurred images and quantitative comparison of SSIM, UQI, BRISQUE and NIQE quality measures.

\begin{tabular}{|c|c|c|c|c|c|c|c|c|c|c|}
\hline \multirow[b]{2}{*}{ Image } & \multirow[b]{2}{*}{ Original Sigma } & \multirow[b]{2}{*}{ Estimated Sigma } & \multicolumn{2}{|r|}{ MSSIM } & \multicolumn{2}{|r|}{ UQI } & \multicolumn{2}{|r|}{ BRISQUE } & \multicolumn{2}{|r|}{ NIQE } \\
\hline & & & Blurred & Proposed Scheme & Blurred & Proposed Scheme & Blurred & Proposed Scheme & Blurred & Proposed Scheme \\
\hline Img-01 & 1.63 & 1.85 & 0.85 & 0.90 & 0.99 & 0.99 & 56.69 & 18.28 & 7.28 & 8.40 \\
\hline Img-02 & 4.98 & 4.76 & 0.73 & 0.81 & 0.95 & 0.97 & 77.15 & 70.69 & 10.84 & 7.97 \\
\hline Img-03 & 5.13 & 5.26 & 0.62 & 0.68 & 0.94 & 0.96 & 79.32 & 73.12 & 11.85 & 9.05 \\
\hline Img-04 & 3.88 & 4.05 & 0.78 & 0.86 & 0.97 & 0.98 & 72.87 & 55.30 & 10.64 & 7.65 \\
\hline Img-05 & 0.71 & 0.68 & 0.88 & 0.95 & 0.99 & 1.00 & 25.45 & 19.06 & 3.80 & 3.88 \\
\hline Img-06 & 0.94 & 1.21 & 0.97 & 0.88 & 1.00 & 1.00 & 52.71 & 38.15 & 7.00 & 7.21 \\
\hline Img-07 & 2.07 & 2.18 & 0.52 & 0.74 & 0.94 & 0.97 & 61.24 & 40.69 & 7.40 & 7.76 \\
\hline Img-08 & 2.63 & 2.50 & 0.69 & 0.80 & 0.95 & 0.97 & 67.06 & 34.43 & 9.10 & 6.99 \\
\hline Img-09 & 3.45 & 3.32 & 0.67 & 0.76 & 0.97 & 0.98 & 67.54 & 54.20 & 10.28 & 6.86 \\
\hline Img-10 & 2.44 & 2.24 & 0.75 & 0.86 & 0.97 & 0.98 & 64.76 & 36.55 & 8.56 & 6.45 \\
\hline Img-11 & 1.64 & 1.82 & 0.75 & 0.88 & 0.98 & 0.99 & 54.33 & 26.65 & 6.56 & 6.59 \\
\hline Img-12 & 0.98 & 0.99 & 0.93 & 0.94 & 0.99 & 1.00 & 47.88 & 11.99 & 5.48 & 3.85 \\
\hline Img-13 & 1.38 & 1.53 & 0.89 & 0.96 & 0.99 & 0.99 & 45.66 & 24.88 & 6.66 & 5.94 \\
\hline Img-14 & 3.13 & 3.26 & 0.62 & 0.80 & 0.93 & 0.97 & 72.01 & 49.99 & 9.79 & 6.85 \\
\hline Img-15 & 4.18 & 4.13 & 0.79 & 0.81 & 0.97 & 0.98 & 80.98 & 58.73 & 10.61 & 8.11 \\
\hline Img-16 & 5.47 & 5.39 & 0.45 & 0.55 & 0.93 & 0.95 & 82.44 & 70.65 & 10.77 & 9.02 \\
\hline
\end{tabular}

Table 2. PSF parameter estimation for out-of-focus blurred images and quantitative comparison of SSIM, UQI, BRISQUE and NIQE quality measures.

\begin{tabular}{|c|c|c|c|c|c|c|c|c|c|c|}
\hline \multirow[b]{2}{*}{ Image } & \multirow[b]{2}{*}{ Original Radius } & \multirow[b]{2}{*}{ Estimated Radius } & \multicolumn{2}{|r|}{ MSSIM } & \multicolumn{2}{|r|}{ UQI } & \multicolumn{2}{|r|}{ BRISQUE } & \multicolumn{2}{|r|}{ NIQE } \\
\hline & & & Blurred & Proposed Scheme & Blurred & Proposed Scheme & Blurred & Proposed Scheme & Blurred & Proposed Scheme \\
\hline Img-01 & 7 & 6.84 & 0.7 & 0.81 & 0.96 & 0.98 & 69.52 & 27.22 & 10.24 & 4.32 \\
\hline Img-02 & 8.85 & 9.2 & 0.7 & 0.76 & 0.94 & 0.97 & 76.69 & 44.17 & 10.42 & 5.34 \\
\hline Img-03 & 10 & 9.4 & 0.59 & 0.7 & 0.93 & 0.95 & 77.68 & 39.09 & 11.99 & 4.97 \\
\hline Img-04 & 11.5 & 11.47 & 0.69 & 0.84 & 0.93 & 0.97 & 76.05 & 32.21 & 12.35 & 5.55 \\
\hline Img-05 & 1 & 0.74 & 0.95 & 0.97 & 1 & 1 & 22.08 & 12.82 & 3.29 & 2.7 \\
\hline Img-06 & 2.5 & 2.46 & 0.97 & 0.93 & 1 & 1 & 60.49 & 14.96 & 8.15 & 5.54 \\
\hline Img-07 & 4 & 3.91 & 0.48 & 0.83 & 0.93 & 0.98 & 57.52 & 10.78 & 6.92 & 3.77 \\
\hline Img-08 & 5.5 & 4.72 & 0.64 & 0.59 & 0.94 & 0.94 & 68.54 & 29.01 & 9.56 & 5.19 \\
\hline Img-09 & 13 & 12.58 & 0.63 & 0.68 & 0.96 & 0.97 & 67.17 & 39.76 & 10.84 & 4.61 \\
\hline Img-10 & 14.5 & 14.14 & 0.63 & 0.61 & 0.93 & 0.95 & 73.93 & 45.1 & 12.12 & 5.31 \\
\hline Img-11 & 16 & 15.27 & 0.51 & 0.58 & 0.93 & 0.95 & 76.35 & 43.96 & 11.36 & 5.2 \\
\hline Img-12 & 17.5 & 18.74 & 0.73 & 0.79 & 0.96 & 0.98 & 77.13 & 37.51 & 11.19 & 4.75 \\
\hline Img-13 & 19 & 18.82 & 0.4 & 0.7 & 0.86 & 0.95 & 80.4 & 45.59 & 11.93 & 5.96 \\
\hline Img-14 & 20.5 & 20.03 & 0.42 & 0.65 & 0.82 & 0.93 & 83.63 & 46.97 & 11.43 & 5.83 \\
\hline Img-15 & 22 & 21.58 & 0.68 & 0.64 & 0.93 & 0.93 & 86.56 & 56.11 & 12.76 & 7.12 \\
\hline Img-16 & 23.5 & 22.89 & 0.38 & 0.47 & 0.89 & 0.91 & 84.8 & 57.47 & 11.57 & 7.85 \\
\hline
\end{tabular}

Table 3. PSF parameter estimation for motion blurred images and quantitative comparison of SSIM and UQI quality measures.

\begin{tabular}{|c|c|c|c|c|c|c|c|c|c|c|c|c|c|c|}
\hline Image & Original Length & Estimated Length & Blurred & Qi Shan & Proposed Scheme & Blurred & Qi Shan & Proposed Scheme & Blurred & Qi Shan & Proposed Scheme & Blurred & Qi Shan & Proposed Scheme \\
\hline Img-01 & 7 & 8 & 0.92 & 0.867 & 0.944 & 0.99 & 0.984 & 0.992 & 34.759 & 34.759 & 24.062 & 6.364 & 5.663 & 5.989 \\
\hline Img-02 & 8 & 8 & 0.875 & 0.894 & 0.926 & 0.985 & 0.986 & 0.992 & 64.961 & 64.961 & 36.537 & 7.059 & 5.146 & 3.913 \\
\hline Img-03 & 9 & 9 & 0.757 & 0.81 & 0.893 & 0.975 & 0.977 & 0.987 & 56.472 & 56.472 & 42.72 & 6.477 & 5.257 & 4.221 \\
\hline Img-04 & 10 & 10 & 0.859 & 0.818 & 0.926 & 0.982 & 0.979 & 0.993 & 65.95 & 65.95 & 38.149 & 8.986 & 5.663 & 4.379 \\
\hline Img-05 & 11 & 12 & 0.74 & 0.73 & 0.828 & 0.983 & 0.974 & 0.987 & 52.707 & 52.707 & 44.811 & 8.393 & 5.916 & 4.311 \\
\hline Img-06 & 12 & 12 & 0.924 & 0.89 & 0.921 & 0.991 & 0.99 & 0.996 & 67.186 & 67.186 & 21.621 & 9.369 & 6.35 & 4.467 \\
\hline Img-07 & 13 & 13 & 0.536 & 0.616 & 0.81 & 0.928 & 0.926 & 0.972 & 39.975 & 39.975 & 24.533 & 7.172 & 7.687 & 5.626 \\
\hline Img-08 & 14 & 13 & 0.775 & 0.86 & 0.858 & 0.965 & 0.977 & 0.978 & 39.233 & 39.233 & 35.9 & 7.278 & 8.694 & 6.288 \\
\hline Img-09 & 15 & 14 & 0.725 & 0.76 & 0.828 & 0.972 & 0.969 & 0.98 & 27.367 & 27.367 & 26.921 & 7.151 & 5.484 & 4.77 \\
\hline Img-10 & 16 & 15 & 0.726 & 0.794 & 0.826 & 0.958 & 0.966 & 0.974 & 26.157 & 26.157 & 24.412 & 7.265 & 4.84 & 4.844 \\
\hline Img-11 & 17 & 17 & 0.572 & 0.53 & 0.804 & 0.942 & 0.942 & 0.978 & 13.188 & 13.188 & 53.311 & 7.936 & 6.072 & 3.844 \\
\hline Img-12 & 18 & 19 & 0.778 & 0.769 & 0.827 & 0.975 & 0.974 & 0.987 & 27.514 & 27.514 & 26.164 & 9.074 & 5.335 & 3.992 \\
\hline Img-13 & $\begin{array}{l}10 \\
19\end{array}$ & 19 & 0.513 & 0.718 & 0.806 & 0.919 & 0.962 & 0.976 & 26.608 & 26.608 & $\begin{array}{l}20.104 \\
46.489\end{array}$ & 9.244 & 6.058 & 4.449 \\
\hline Img-14 & 20 & 21 & 0.551 & 0.673 & 0.771 & 0.894 & 0.945 & 0.967 & 25.011 & 25.011 & 45.833 & 8.099 & 5.232 & 4.312 \\
\hline Img-15 & 21 & 21 & 0.755 & 0.679 & 0.587 & 0.969 & 0.956 & 0.962 & 58.33 & 58.33 & 55.449 & 8.397 & 6.492 & 5.462 \\
\hline Img-16 & 22 & 24 & 0.504 & 0.551 & 0.674 & 0.934 & 0.932 & 0.963 & 49.092 & 49.092 & 45.294 & 6.07 & 5.92 & 5.615 \\
\hline
\end{tabular}

Table 4. BRISQUE and NIQE quality measures of the deblurred images.

\begin{tabular}{lrrrrrrrr}
\hline Image & \multicolumn{4}{c}{ BRISQUE } & \multicolumn{5}{c}{ NIQE } \\
\hline & Original & Shan & Cho & Proposed & Original & Shan & Cho & Proposed \\
\hline LABEL & 56.08 & 52.00 & 48.811 & 54.07 & 7.11 & 8.1 & 7.984 & 7.68 \\
DIP-BOOK & 29.77 & 40.21 & 33.302 & 45.68 & 5.78 & 6.31 & 5.078 & 6.51 \\
MATLAB-BOOK & 5.48 & 28.84 & 24.490 & 48.55 & 5.76 & 4.07 & 8.277 & 6.31 \\
BUILDINGS & 39.85 & 41.17 & 46.976 & 39.58 & 7.01 & 6.41 & 6.117 & 6.85 \\
\hline
\end{tabular}


spectral kurtosis is proposed to estimate uniform blur for a parametric form of PSF. A steepest descent optimization scheme is employed to optimize the blur parameter in the direction of maximum absolute kurtosis of the deblurred image. The proposed method operates on single image and is simple, efficient and easy to implement. The method is applicable to various types of blurs such as Gaussian, motion and out-of-focus. Experiments on both artificial and real blurred images have shown the capability and marked improvement of the scheme over the existing methods, in terms of both visual perception and a range of quality measures.

\section{APPENDICES}

\section{A. MOTION BLUR}

Without loss of generality, the linear motion modelled is the result of translation in the horizontal direction. In order to simplify the search for blur parameters with the steepest descent scheme, one can rotate the image until the blur can be assumed horizontal. This simplifies the case from two parameters, length $(L)$ and angle $(\varphi)$ of blur to only the length that needs to be optimized. The OTF for motion blur according to Biemond et al. (1990) is given by

$$
\hat{H}(i, j)=\frac{1}{L+1} \mathrm{e}^{-k i \pi L} \frac{\sin (\pi(L+1) i))}{\sin (\pi i)}, \quad \forall j .
$$

Differentiating the OTF with respect to parameter $L$ of the OTF:

$$
\begin{aligned}
& \frac{\partial}{\partial \lambda} \hat{H}(i, j)= \frac{\partial}{\partial L} \hat{H}(i, j) \\
&= \frac{\partial}{\partial L}\left(\frac{1}{L+1} \mathrm{e}^{-k i \pi L} \frac{\sin (\pi(L+1) i))}{\sin (\pi i)}\right) \\
&= \frac{-1}{(L+1)} \hat{H}(i, j)-k \pi i \hat{H}(i, j) \\
&\quad+(\pi i) \cot (\pi(L+1) i)) \hat{H}(i, j) \\
&= \hat{H}(i, j)\left(-\frac{1}{L+1}-k \pi i\right. \\
&\quad+\pi i \cot (\pi(L+1) i)) .
\end{aligned}
$$

\section{B. OUT-OF-FOCUS BLUR}

The OTF for out-of-focus blur (Biemond et al., 1990) is given by,

$$
\hat{H}(i, j)=\frac{J_{1}(R p)}{R p}, \quad p^{2}=i^{2}+j^{2},
$$

where $J_{1}$ is the first order Bessel function. The Bessel function can be approximated by an exponential for sufficiently large data (Olver et al., 2010):

$$
\begin{aligned}
& \hat{H}(i, j)=\frac{\mathrm{e}^{R p}}{\sqrt{2 \pi R p}} \\
& \frac{\partial}{\partial R} \hat{H}(i, j)= \frac{\partial}{\partial R}\left(\frac{\mathrm{e}^{R p}}{\sqrt{2 \pi R p}}\right) \\
&= \mathrm{e}^{R p} \frac{\partial}{\partial R}\left(\frac{1}{\sqrt{2 \pi R p}}\right) \\
&+\left(\frac{1}{\sqrt{2 \pi R p}}\right) \frac{\partial}{\partial R}\left(\mathrm{e}^{R p}\right) \\
&=(2 \pi p) \mathrm{e}^{R p} \frac{(-1 / 2)}{\sqrt{2 \pi R p}}+\left(\frac{\mathrm{e}^{R p}}{\sqrt{2 \pi R p}}\right)(R p) \\
&= \frac{\mathrm{e}^{R p}}{\sqrt{2 \pi R p}}(-\pi p+R p) \\
&= \hat{H}(i, j)(-\pi p+R p) .
\end{aligned}
$$

\section{REFERENCES}

Banham MR, Katsaggelos AK (1997). Digital image restoration. IEEE Signal Pro Mag 14:24-41.

Biemond J, Lagendijk RL, Mersereau RM (1990). Iterative methods for image deblurring. Proc IEEE 78:856-83.

Brunet D, Vrscay ER, Wang Z (2012). On the mathematical properties of the structural similarity index. IEEE $T$ Image Process 21:1488-99.

Chan TF, Wong CK (1998). Total variation blind deconvolution. IEEE T Image Process 7:370-5.

Chen D, Cheng L (2011). Alternative minimisation algorithm for non-local total variational image deblurring. IET Image Process 4:353-64.

Cho S, Lee S (2009). Fast motion deblurring. ACM T Graphic 28:145.

Gao Y, Rehman A, Wang Z (2011). CW-SSIM based image classification. In: Proc 18th IEEE Int Conf Image Process 1249-52.

Gupta A, Joshi N, Zitnick CL, Cohen M, Curless B (2010). Single image deblurring using motion density functions. In: Daniilidis K, Maragos P, Paragios N, eds. Proc 11th Eur Conf Comput Vision (ECCV 2010). Lect Not Comput Sci 6311:171-84.

Harmeling S, Scholkopf B, Schuler CJ, Hirsch M (2011). Fast removal of non-uniform camera shake. In: Proc 2011 IEEE Int Conf Comput Vision (ICCV 2011) 46370. 
Khan A, Yin H (2011). Spectral non-gaussianity for blind image deblurring. In: Yin H, Wang W, Rayward-Smith V, eds. Proc 12th Int Conf Intel Data Eng Autom Learn (IDEAL 2011). Lect Not Comput Sci 6936:144-51.

Kundur D, Hatzinakos D (1996). Blind image deconvolution. IEEE Signal Proc Mag 13:43-64.

Lagendijk RL, Biemond J (2009). Basic methods for image restoration and identification. In: Bovik A, ed. The essential guide to image processing, 2nd Ed. San Diego: Academic Press, 323-48.

Lagendijk RL, Biemond J, Boekee DE (1988). Regularized iterative image-restoration with ringing reduction. IEEE T Acoust Speech 36:1874-88.

Lagendijk RL, Tekalp AM, Biemond J (1990). Maximumlikelihood image and blur identification - a unifying approach. Opt Eng 29:422-35.

Lu C (2012). Image restoration and decomposition using nonconvex non-smooth regularisation and negative hilbertsobolev norm. IET Image Process 6:706-16.

Mittal A, Moorthy AK, Bovik AC (2011). Brisque software release.

Mittal A, Moorthy AK, Bovik AC (2012a). Niqe software release.

Mittal A, Moorthy AK, Bovik AC (2012b). No-reference image quality assessment in the spatial domain. IEEE T Image Process 21:4695-708.

Mittal A, Soundararajan R, Bovik AC (2013). Making a "completely blind" image quality analyzer. IEEE Signal Proc Let 20:209-12.

Nexus D (2010). Desktop nexus wallpaper image database.

Olver FWJ, Lozier DW, Boisvert RF, Clark CW, eds (2010). NIST Handbook of mathematical functions. New York: Cambridge University Press.

Oppenheim A, Schafer RW, Stockham TG (1968). Nonlinear filtering of multiplied and convolved signals. Proc IEEE 56:1264-85.
Rehman A, Wang Z (2011). SSIM-based non-local means image denoising. In: Proc 18th IEEE Int Conf Image Process 217-20.

Richardson W (1972). Bayesian-based iterative method of image restoration. J Opt Soc Am 62:55-9.

Shan Q, Jia J, Agarwala A (2008). High-quality motion deblurring from a single image. ACM T Graphic 27:73.

Sondhi MM (1972). Image restoration - removal of spatially invariant degradations. Proc IEEE 60:842-53.

Szolgay D, Szirányi T (2011). Optimal stopping condition for iterative image deconvolution by new orthogonality criterion. Electron Lett 47:442-4.

Wang Z, Bovik AC (2002). A universal image quality index. IEEE Signal Proc Let 9:81-4.

Wang Z, Bovik AC, Sheikh HR, Simoncelli EP (2004). Image quality assessment: From error visibility to structural similarity. IEEE T Image Process 13:600-12.

Wang Z, Li Q (2011). Information content weighting for perceptual image quality assessment. IEEE $\mathrm{T}$ Image Process 20:1185-98.

Whyte O, Sivic J, Zisserman A (2011). Deblurring shaken and partially saturated images. Int $\mathrm{J}$ Comput Vis 110:185.

Wiggins RA (1978). Minimum entropy deconvolution. Geoexploration 16:21-35.

Yang S, Liu B (2011). Image deblurring using weighted total variation regularisation for half-quadratic model. Electron Lett 47:1225-7.

Yin HJ, Hussain I (2008). Independent component analysis and non-Gaussianity for blind image deconvolution and deblurring. Integr Comput Aid Eng 15:219-28.

Yuquan X, Lu W, Xiyuan H, Silong P (2013). Single-image blind deblurring for non-uniform camera-shake blur. In: Lee KM, Matsushita Y, Rehg JM, Hu Z, eds. Proc 11th Asian Conf Comput Vision (ACCV 2012). Lect Not Comput Sci 7726:336-48. 